\title{
ON THE ROLE OF CYCLIC NUCLEOTIDES IN THE TRANSMITTER CHOICE MADE BY CULTURED SYMPATHETIC NEURONS ${ }^{1}$
}

\author{
PATRICIA A. WALICKE AND PAUL H. PATTERSON ${ }^{2}$
}

Department of Neurobiology, Harvard Medical School, Boston, Massachusetts 02115

\begin{abstract}
Previous investigations have established that electrical activity or chronic depolarization influences the development of neonatal rat sympathetic neurons in dissociated cell culture. Depolarization reduces their ability to respond to a cholinergic inducing factor produced by non-neuronal cells, allowing normal adrenergic differentiation to proceed (Walicke, P., R. Campenot, and P. Patterson (1977) Proc. Natl. Acad. Sci. U. S. A. 74: 5767-5771). The present study examines whether the developmental effects of depolarization are mediated through cyclic nucleotides. Addition of dibutyryl cAMP, dibutyryl cGMP, adenosine, prostaglandin $\mathrm{E}_{1}$, and cholera toxin all raise neuronal cyclic nucleotide levels and qualitatively mimic the developmental effects of depolarization. However, the quantitative decrease in acetylcholine production caused by these cyclic nucleotide agents is much smaller than that caused by depolarization. Short (48-hr) exposures to the cyclic nucleotide derivatives do not alter transmitter synthesis, indicating that long term developmental changes are involved. Chronic depolarization with elevated $\mathrm{K}^{+}$increases neuronal cAMP 2 -fold but has little effect on cGMP. The increase in cAMP is maintained during several weeks of depolarization and is present as early as the 3rd day in vitro, preceding the significant alterations in adrenergic and cholinergic differentiation. Exposure to $2 \mathrm{mM}$ theophylline also increases neuronal cAMP, but, in contrast to the other agents, it enhances cholinergic differentiation. In combination with elevated $\mathrm{K}^{+}$, theophylline further increases neuronal cAMP but still favors cholinergic differentiation. Thus, although cAMP satisfies some criteria for being the second messenger in the developmental effects of depolarization, several findings are inconsistent with the nucleotide playing a central role: $(i)$ Depolarization has much larger effects on transmitter choice than the cyclic nucleotide agents and (ii) theophylline can uncouple cyclic nucleotide levels from the developmental events.
\end{abstract}

Cultures of dissociated sympathetic neurons from superior cervical ganglia of newborn rats have been studied extensively in order to delineate signals influencing neuronal development. When grown alone in culture, these neurons can complete the course of adrenergic differentiation that they had begun in vivo. However, when grown in the presence of a variety of non-neuronal cell types, or in medium conditioned by such non-neuronal cells (CM), the sympathetic neurons can be induced to express cholinergic properties. Concomitant with the increase in cholinergic function is a decrease in the ability to synthesize catecholamine (CA) (Patlerson, 1978; Bunge et al., 1978). Analysis of single neurons showed that the vast majority of these cells can be induced to

\footnotetext{
' This work was supported by the National Institute of Neurological and Communicative Disorders and Stroke, the Dysautononia Foundation, and the American and Massachusetts Heart Associations. Dr. Walicke was a United States Public Health Service predoctoral fellow. Preliminary reports of this work have appeared previously (Walicke, P. A., and P. H. Patterson (1978) Soc. Neurosci. Abstr. 4: 129; Walicke, P. A., and P. H. Patterson (1979) Soc. Neurosci. Abstr. 5: 183).

${ }^{2}$ To whom correspondence should be addressed.
}

become cholinergic (Reichardt and Patterson, 1977). This observation was surprising, since most sympathetic neurons in vivo become adrenergic. It seemed possible that there were factors missing from the culture system which, in vivo, might counteract the response of the neurons to the cholinergic factor.

It was observed that chronic depolarization with 20 $\mathrm{mM} \mathrm{K} \mathrm{K}^{+}$or veratridine (an alkaloid which opens voltagedependent $\mathrm{Na}^{+}$channels) directed the neurons to complete adrenergic differentiation, even in the presence of CM (Walicke et al., 1977). The effect of these agents was very striking; the acetylcholine to catecholamine (ACh/ CA) ratio (used as an index of the proportion of neurons undergoing cholinergic or adrenergic differentiation) $w$ is lowered as much as 500 -fold. The change was almost entirely due to suppression of $\mathrm{ACh}$ synthesis, although $20 \mathrm{mM} \mathrm{K}^{+}$, in the absence of $\mathrm{CM}$, appeared to enhance adrenergic differentiation somewhat, roughly doubling the content of $\mathrm{CA} /$ neuron and increasing the proportion of small granular (adrenergic) vesicles (Landis, 1980). Direct electrical stimulation at a frequency of $1 \mathrm{~Hz}$ also markedly decreased the response to $\mathrm{CM}$; thus, the effects 
of chronic depolarization appear to be the same as those of normal neuronal activity. The effect of depolarization or activity on transmitter choice was a specific one in that depolarization did not alter neuronal survival or overall growth, as monitored by total protein content (Walicke et al., 1977). The alterations in transmitter choice were interpreted as indicating that electrical activity acts as a determination or maturation signal, fixing the neurons in their initial adrenergic mode.

Little is known about how electrical activity may lead to long term alterations in cellular metabolism. It was of interest, therefore, to use this system to investigate the intracellular mechanism through which activity has its effect on development. In adult adrenergic tissues, increased synaptic stimulation leads to induction of tyrosine hydroxylase (TH) and dopamine $\beta$-hydroxylase (Zigmond and Chalazonitis, 1979), an effect somewhat analogous to that under study here. Cyclic adenosine $3^{\prime}: 5^{\prime}-$ monophosphate (cAMP) has been suggested to serve as the second messenger in trans-synaptic induction in the adrenal medulla (Guidotti and Costa, 1977). Derivatives of cAMP increase the levels of tyrosine hydroxylase and dopamine $\beta$-hydroxylase and CA content in neuroblastoma cell lines (Prasad, 1975; Waymire et al., 1978) and organ-cultured ganglia (Goodman et al., 1974; Keen and McLean, 1974; Mackay and Iversen, 1972). Cyclic AMP, therefore, seemed a reasonable candidate for the second messenger in the developmental effects of depolarization.

If cAMP is playing such a role, then one would expect that (i) exogenous cyclic nucleotides and effectors which increase cellular cyclic nucleotide levels should be able to mimic the developmental effects of activity, (ii) increases in activity should lead to increases in cAMP, (iii) the increase in cAMP should precede detectable changes in transmitter content, (iv) phosphodiesterase inhibitors should increase the developmental effects of activity, and $(v)$ agents which antagonize the effects of activity on transmitter choice should block its effects on cAMP. These criteria are examined in the experiments presented in this paper.

\section{Materials and Methods}

Superior cervical ganglia were dissected and cultured as previously described (Hawrot and Patterson, 1978). All cultures were plated initially into normal $\mathrm{L} 15-\mathrm{CO}_{2}$ medium; pharmacological agents were added with the first change of medium on day 2. All of the medium needed for an experiment was mixed with the appropriate drugs at the time of the first feeding, and aliquots of appropriate size for one feeding were made up and frozen until needed. Cholera toxin was purchased from Schwarz/Mann. RO 20-1724 was a gift of Roche Pharmaceuticals. All other chemicals were obtained from Sigma.

Conditioned medium. CM was made by incubation on monolayer cultures of rat heart or skeletal muscle cells as previously described (Patterson and Chun, 1977a). Instead of CM, some neuronal cultures were incubated with a CM preparation purified by ammonium sulfate precipitation (M. Weber, unpublished technique). For this, $2.5 \mathrm{mM}$ EGTA (ethylene glycol bis( $\beta$-aminoethyl ether)- $N, N^{\prime}$-tetra-acetic acid) and $0.3 \mathrm{~mm}$ phenylmethylsulfonyl flouride were added to pooled batches of Meth- ocel-free $\mathrm{CM}$ at $4^{\circ} \mathrm{C}$. The $\mathrm{pH}$ was held at about 7.3 with $\mathrm{NH}_{4} \mathrm{OH}$, observing the color of the indicator dye in the medium. Ammonium sulfate was added to $50 \%$ saturation and the CM was centrifuged at $4000 \times \mathrm{g}$ for $30 \mathrm{~min}$. The precipitate was discarded, ammonium sulfate was added to saluration, and the CM was centrifuged at $4000 \times \mathrm{g}$ for $60 \mathrm{~min}$. The precipitate was resuspended in a minimal volume of $\mathrm{H}_{2} \mathrm{O}$ and placed in Spectropore dialysis tubing $(12,000$ to 14,000 daltons cutoff). Dialysis tubing was prepared by boiling twice for $15 \mathrm{~min}$ in glass-distilled $\mathrm{H}_{2} \mathrm{O}$, followed by autoclaving. Dialysis was for 2 days at $4^{\circ} \mathrm{C}$ against three 1-liter changes of distilled $\mathrm{H}_{2} \mathrm{O}$. The retentate was stored at $-20^{\circ} \mathrm{C}$ except for an aliquot which was incubated at $37^{\circ} \mathrm{C}$ to test for sterility. Sterile technique was employed whenever possible, and the resulting material was usually sterile. The dialyzed ammonium sulfate fraction was added to give medium containing the equivalent of 60 to $100 \% \mathrm{CM}$, and it never constituted more than $10 \%$ of the final volume of the medium.

Assays. Neuronal transmitter synthesis was determined by incubation with $\left[{ }^{3} \mathrm{H}\right]$ tyrosine and $\left[{ }^{3} \mathrm{H}\right]$ choline for $4 \mathrm{hr}$ and electrophoretic separation of the products as previously described (Patterson and Chun, 1977a). All incubations were performed in normal $\mathrm{L} 15-\mathrm{CO}_{2}$ medium (in the absence of phosphodiesterase inhibitors, cyclic nucleotide derivatives, depolarizing agents, and $\mathrm{CM}$ ).

Cyclic nucleotides were assayed with cAMP and cGMP (cyclic guanosine $3^{\prime}: 5^{\prime}$-monophosphate) radioimmunoassay kits purchased from New England Nuclear using the acetylated version with a sensitivity of $5 \mathrm{fmol}$. The day before assay, Methocel-free $\mathrm{L} 15-\mathrm{CO}_{2}$ medium with all other additives and pertinent pharmacological agents was placed in the same incubator as the cultures to reach the same temperature and $\mathrm{pH}$. Before harvesting, cultures were rinsed with $1 \mathrm{ml}$ of this medium and then incubated $10 \mathrm{~min}$ in another $\mathrm{ml}$. For cultures grown in dibutyryl cAMP (dbcAMP) or dibutyryl cGMP (dbcGMP), the cyclic nucleotide was omitted from the rinse, and the cultures were rinsed five times with $1 \mathrm{ml}$ to remove the added nucleotide. The medium was removed, the culture was drained carefully with a cottontipped applicator, and the neurons were carefully scraped free of the collagen film with a small surgical blade. Two cultures were combined for each sample. They were homogenized in $100 \mu$ l of $6 \%$ trichloroacetic acid in a microhomogenizer (Micro or Kontes) which then was rinsed twice with $100 \mu \mathrm{l}$ each of acid. 'The combined samples were centrifuged for $30 \mathrm{~min}$ at high speed in a Sorvall GLC-1 centrifuge at $4^{\circ} \mathrm{C}$. The supernatant fraction was collected for cyclic nucleotide assay. The protein precipitate was redissolved and assayed by the method of Lowry (Lowry et al., 1951) using bovine serum albumin as a standard. The supernatant fractions were washed four times with $1.5 \mathrm{ml}$ of water-saturated ether. They were incubated briefly in a warm water bath under a stream of air to remove the last of the ether and then frozen and lyophilized. The samples were resuspended in $200 \mu \mathrm{l}$ of acetate buffer $(0.05 \mathrm{M}, \mathrm{pH} 6.2) ; 100 \mu$ l was used for the cGMP assay and 50 to $100 \mu$ for the cAMP assay. Recoveries of the $\left[{ }^{3} \mathrm{H}\right] \mathrm{cAMP}$ and $\left[{ }^{3} \mathrm{H}\right] \mathrm{cGMP}$ added to the assays were 30 to $40 \%$. External standard curves were run with each assay. 


\section{Results}

Effects of cyclic nucleotide derivatives and effectors on neuronal development. Before examining the influence of cyclic nucleotides on neuronal transmitter choice, it was necessary to consider the influence of these agents on cell survival and growth. Neurons were plated into L15- $\mathrm{CO}_{2}$ growth medium and then exposed to phosphodiesterase inhibitors and cyclic nucleotide derivatives from the 2nd day in vitro onward. Counts of neuronal somata on the 18th day demonstrated no significant alterations in cell survival. Furthermore, transmitter assay on day 20 revealed no alteration in the amount of CA synthesized and stored per neuron attributable to the cyclic nucleotides, though $20 \mathrm{~mm} \mathrm{\textrm {K } ^ { + }}$ roughly doubled this capacity (Table I). Protein content was not examined in this experiment but was routinely determined in cultures assayed for cyclic nucleotide content. Neither dbcAMP nor dbcGMP significantly altered the amount of protein per culture (see below).

To examine the effects of butyryl derivatives of both cAMP and cGMP on cholinergic induction, neurons were grown for 20 days in the presence of the additives with $60 \% \mathrm{CM}$ also present for the last 10 days (Table II). Control cultures responded well to the $\mathrm{CM}$, with a final $\mathrm{ACh} / \mathrm{CA}$ ratio of 56.0. Neurons grown in either mono- or dibutyryl cAMP had roughly 3- to 4-fold lower $\mathrm{ACh} / \mathrm{CA}$ ratios than controls, primarily due to lower induction of ACh synthesis. Neurons treated with the cGMP derivatives responded similarly to those treated with the cAMP derivatives.

In earlier experiments with $\mathrm{K}^{+}$, veratridine, and electrical stimulation, decreases in the $\mathrm{ACh} / \mathrm{CA}$ ratio of more than 100 -fold were often seen (Walicke et al., 1977). The 3- to 5-fold decreases seen with the cyclic nucleotide agents were small by comparison. Efforts were made to optimize the effects of the cyclic nucleotide derivatives by (i) testing native and 8-monobutyryl derivatives, (ii) testing dibutyryl derivatives with and without phosphodiesterase inhibitors, and (iii) performing dose-response curves of dbcAMP and dbcGMP over the range of 0.1 to $3.0 \mathrm{~mm}$. The largest decrease in the $\mathrm{ACh} / \mathrm{CA}$ ratio attributable to cyclic nucleotide derivatives was 15 -fold. (It should be noted that, although there is always a large difference between the transmitter ratios of controls and CM-treated cultures, the absolute values of the ratios vary from one experiment to the next. One possible reason for this could be that the many preparations of $\mathrm{CM}$ used in these studies varied in their potency for inducing ACh synthesis.)

To ascertain whether the effects of the butyryl analogues were due to the nucleotides, butyrate controls were performed (Table III). Butyrate is somewhat toxic for many growing cells (Waymire et al., 1978), and treated neurons were smaller when viewed with the phase microscope and had lower total transmitter synthesis than controls. Interestingly, neurons exposed to $1 \mathrm{~mm}$ butyrate and $\mathrm{CM}$ were nearly purely cholinergic, with an $\mathrm{ACh} / \mathrm{CA}$ ratio more than 100 -fold higher than those grown in $\mathrm{CM}$ alone. The major change in the ratio is due to suppression of CA synthesis to barely detectable levels. ACh synthesis also is depressed slightly, which may reflect the general decrease in neuronal growth. Since the butyryl cyclic nucleotide derivatives were used in the presence of phos-
TABLE I ${ }^{3}$

Effects of cyclic nucleotide derivatives on neuronal survival and $C A$ synthesis

Neuronal number was determined on day 18, and transmitter synthesis on day 20. Each group contained triplicate cultures. All figures are the mean \pm SEM in this and the following tables.

\begin{tabular}{llc}
\hline \multicolumn{1}{c}{ Additives } & Neuronal Number & $\mathrm{CA}$ \\
\hline & & $\mathrm{fmol} / \mathrm{cell}$ \\
None & $3775 \pm 1138$ & $2.26 \pm 0.41$ \\
$20 \mathrm{mM} \mathrm{K}^{+}$ & $2156 \pm 788$ & $4.73 \pm 0.46$ \\
$1 \mathrm{~mm}$ mbcAMP + R & $3000 \pm 1679$ & $2.84 \pm 0.01$ \\
$1 \mathrm{~mm} \mathrm{dbcAMP} \mathrm{+} \mathrm{R}$ & $4190 \pm 297$ & $2.60 \pm 0.44$ \\
$1 \mathrm{~mm} \mathrm{8BrcAMP} \mathrm{+} \mathrm{R}$ & $2846 \pm 532$ & $2.82 \pm 0.98$ \\
$1 \mathrm{~mm}$ mbcGMP + I & $3140 \pm 28$ & $2.22 \pm 0.56$ \\
$1 \mathrm{~mm} \mathrm{dbcGMP} \mathrm{+} \mathrm{I}$ & $3200 \pm 116$ & $3.32 \pm 0.60$ \\
$\mathrm{R}$ & $3080 \pm 160$ & $2.37 \pm 0.36$ \\
I & $3170 \pm 75$ & $1.88 \pm 0.26$ \\
\hline
\end{tabular}

phodiesterase inhibitors, butyrate also was examined in their presence, and they had no significant effect on transmitter synthesis. Thus, butyrate has effects on neuronal development which are the opposite of those of the dibutyryl cyclic nucleotides; that is, it suppresses adrenergic and strongly favors cholinergic development.

Effects of pretreatment and short term incubation with cyclic nucleotide derivatives. Cyclic AMP can activate $\mathrm{TH}$ through a kinase which phosphorylates $\mathrm{TH}$ and converts it to a more active form (Joh et al., 1978; Vulliet et al., 1980). Although interactions between cyclic nucleotides and choline acetyltransferase have not been described, it seemed possible that the responses of neurons exposed to dibutyryl cAMP or cGMP reflect phosphorylation-induced modulations in the activity of existing enzymes rather than changes in enzyme synthesis. If the alterations in transmitter synthesis are due simply to changes in activity of existing enzymes, it should be possible to influence transmitter synthesis by short exposures to cyclic nucleotides. Cultures were grown in CM and exposed to dbcAMP or dbcGMP for the final 2 days before assay (Table IV). Neither nucleotide caused an increase in CA synthesis or a decrease in ACh synthesis during this short exposure. To demonstrate further that the observed changes are due to stable alterations, the converse experiment was performed also. Removal of dbcAMP or dbcGMP and phosphodiesterase inhibitors for 2 days preceding the assay again caused little alteration in the $\mathrm{ACh} / \mathrm{CA}$ ratio or in $\mathrm{ACh}$ or $\mathrm{CA}$ synthesis. Thus, the changes in transmitter synthesis are likely to be stable alterations and not simply modulation of the activities of existing enzymes.

The role of $c G M P$. Previous reports have indicated that cells exposed to exogenously added cyclic nucleotides can adapt to the presence of the additive by increasing their content of the other nucleotide (Prasad and Gilmer, 1974; Zwiller et al., 1977). The present observation that both dbcAMP and dbcGMP decrease cholinergic induction could mean that both cyclic nucleotides have the same effect on transmitter choice. It is also possible, however, that only one of the two cyclic nucleotides is involved and exposure to the other derivative

\footnotetext{
${ }^{3}$ The abbreviations used in the tables are: $8 \mathrm{Br}$, monobutyryl- 8 . bromo; db, dibutyryl; I, $10 \mu \mathrm{M}$ isobutyl methylxanthine; mb, monobutyryl; R, $10 \mu \mathrm{M}$ RO 20-1724.
} 
TABLE II

Effects of cyclic nucleotide derivatives on transmitter choice

Sixty percent CM was present in the medium of all samples from days 11 to 20; cultures were assayed on day 21 . Each group contained triplicate cultures. ACh/CA ratios are the mean of the values computed for three individual cultures. Statistics were computed as an analysis of variance; comparisons were made using the method of Scheffé (1959).

\begin{tabular}{lccr}
\hline \multicolumn{1}{c}{ Additives } & ACh/CA & ACh & pA \\
\hline & & & pmol/culture \\
CM & $56.0 \pm 1.9$ & $240.9 \pm 31.2$ & $4.8 \pm 0.5$ \\
CM + 1 mM mbcAMP + R & $15.0 \pm 0.9^{a}$ & $101.3 \pm 22.6^{a}$ & $6.8 \pm 1.9$ \\
CM + 1 mM dbcAMP + R & $17.7 \pm 2.7^{a}$ & $116.7 \pm 16.7^{a}$ & $6.7 \pm 0.9$ \\
CM + 1 mM mbcGMP + I & $22.5 \pm 4.1^{a}$ & $96.1 \pm 17.4^{a}$ & $4.3 \pm 0.4$ \\
CM + 1 mM dbcGMP + I & $11.2 \pm 2.6^{a}$ & $23.6 \pm 5.4^{a}$ & $2.1 \pm 0.1$ \\
\hline
\end{tabular}

${ }^{a} p<0.05$ compared to control (line 1$)$.

TABLE III

Effects of butyrate on neuronal transmitter choice

All cultures were induced with $50 \% \mathrm{CM}$ and assayed on day 20. Each group contained triplicate cultures.

\begin{tabular}{lccc}
\hline \multicolumn{1}{c}{ Additives } & $\mathrm{ACh} / \mathrm{CA}$ & $\mathrm{ACh}$ & cA \\
\hline & & & pmol/culture \\
$\mathrm{CM}$ & $1.7 \pm 0.8$ & $23.0 \pm 12.0$ & $16.00 \pm 6.40$ \\
$\mathrm{CM}+1 \mathrm{mM}$ butyrate & $293.0 \pm 39.9^{a}$ & $11.3 \pm 0.5$ & $0.04 \pm 0.01^{a}$ \\
$\mathrm{CM}+1 \mathrm{mM}$ butyrate $+\mathrm{R}$ & $201.8 \pm 90.7^{a}$ & $19.1 \pm 3.5$ & $0.10 \pm 0.03^{a}$ \\
$\mathrm{CM}+1 \mathrm{mM}$ butyrate $+\mathrm{I}$ & $242.8 \pm 56.6^{a}$ & $15.0 \pm 2.1$ & $0.07 \pm 0.02^{a}$ \\
\hline
\end{tabular}

$p<0.01$ versus control (line 1 ).

TABLE IV

Effects on transmitter synthesis of short exposure to cyclic nucleotide

All cultures were exposed to $50 \% \mathrm{CM}$ for induction, those in the first experiment from days 10 to 20 and those in the second from days 2 to 14 . Cultures exposed to cAMP were assayed on day 21; those exposed to cGMP on day 15. Each group contained triplicate cultures. In this table, dbcAMP refers to $1 \mathrm{mM}$ dibutyryl cAMP and dbcGMP refers to $1 \mathrm{mM}$ dibutyryl cGMP.

\begin{tabular}{|c|c|c|c|c|}
\hline \multicolumn{5}{|c|}{ Experiment 1} \\
\hline Days 2-18 & Days $18-20$ & $\mathrm{ACh} / \mathrm{CA}$ & $\mathrm{ACh}$ & $\mathrm{CA}$ \\
\hline & & & \multicolumn{2}{|c|}{ pmol/culture } \\
\hline $\mathrm{CM}$ & $\mathrm{CM}$ & $15.6 \pm 2.1$ & $93.6 \pm 10.9$ & $6.1 \pm 0.7$ \\
\hline $\mathrm{CM}$ & $\mathrm{CM}+$ dbcAMP + R & $15.8 \pm 3.0$ & $104.7 \pm 18.1$ & $6.7 \pm 0.8$ \\
\hline $\mathrm{CM}+\mathrm{dbcAMP}+\mathrm{R}$ & $\mathrm{CM}+$ dbcAMP $+\mathrm{R}$ & $3.7 \pm 1.2^{a}$ & $31.5 \pm 9.9$ & $8.5 \pm 0.1$ \\
\hline $\mathrm{CM}+$ dbcAMP $+\mathrm{R}$ & $\mathrm{CM}$ & $5.5 \pm 1.0^{a}$ & $43.0 \pm 7.5$ & $7.9 \pm 0.9$ \\
\hline \multicolumn{5}{|c|}{ Experiment 2} \\
\hline Days 2-12 & Days $12-14$ & & & \\
\hline CM & $\mathrm{CM}$ & $3.2 \pm 0.3$ & $94.7 \pm 9.2$ & $30.1 \pm 8.6$ \\
\hline $\mathrm{CM}$ & $\mathrm{CM}+$ dbcGMP + R & $2.8 \pm 0.1$ & $70.3 \pm 7.3$ & $25.0 \pm 1.9$ \\
\hline $\mathrm{CM}+\mathrm{dbcGMP}+\mathrm{R}$ & $\mathrm{CM}+\mathrm{dbcGMP}+\mathrm{R}$ & $0.8 \pm 0.2^{a}$ & $26.7 \pm 8.1^{a}$ & $30.9 \pm 2.4$ \\
\hline $\mathrm{CM}+\mathrm{dbcGMP}+\mathrm{R}$ & $\mathrm{CM}$ & $0.9 \pm 0.1^{\alpha}$ & $32.1 \pm 2.8^{a}$ & $36.5 \pm 0.6$ \\
\hline
\end{tabular}

${ }^{a} p<0.05$ compared to $\mathrm{CM}$ control (line 1 of Experiment 1 or 2 ).

caused a compensatory increase, yielding deceptive positive results. To investigate whether the sympathetic neurons alter the level of one cyclic nucleotide in response to exposure to the other dibutyryl derivative, the intracellular cyclic nucleotide content was determined (Table V). In this experiment, CM treatment and addition of cyclic nucleotide derivatives affected the ACh/ $\mathrm{CA}$ ratios in accordance with the previous observations.

Determination of cyclic nucleotide content showed that control cultures contained considerably more cAMP than cGMP. Exposure to CM lowered the content of cAMP slightly and increased the content of cGMP slightly. Exposure to $10 \mu \mathrm{M}$ RO 20-1724 caused slight increases in the content of both cAMP and cGMP. Neurons exposed continuously to dbcGMP, either in the presence or absence of CM, contained about twice as much cAMP as controls or about 1.5-fold as much as neurons exposed to RO 20-1724 alone. Neurons which were exposed only to dbcGMP for the last 2 days before assay did not contain increased cAMP. The average content of cGMP in these dbcGMP cultures was 19.8 $\mathrm{fmol} / \mu \mathrm{g}$ of protein, which probably included dbcGMP still present in the medium collected with the neurons but did indicate that the rinsing procedure was efficient. Since the cross-reactivity of cAMP and cGMP in the assays was only $0.05 \%$, the observed increase in cAMP is not likely to be an artifact. On the other hand, neurons grown in dbcAMP, or exposed to it for the last 2 days before assay, did not contain increased cGMP. Neurons grown in dbcAMP and CM, however, did contain nearly 4 -fold more cGMP than controls or 2.5 -fold more than those in CM alone. 
To clarify further whether cAMP, cGMP, or both are involved in the transmitter choice, the effects of cholera toxin, adenosine, and prostaglandin $\mathrm{E}_{1}\left(\mathrm{PGE}_{1}\right)$ on transmitter synthesis and cyclic nucleotide content were examined (Table VI). Each of these effectors lowered the $\mathrm{ACh} / \mathrm{CA}$ ratio 4- to 6 -fold primarily because of a suppression of the increase in ACh synthesis. In cultures not exposed to $\mathrm{CM}$, the effectors suppressed $\mathrm{ACh}$ production to the level of detectability. Neurons exposed to cholera toxin contained nearly 3 -fold more cAMP than controls. In the presence of CM and cholera toxin, cAMP levels were lower but still 3 -fold higher than in CM alone. It is significant that cholera toxin did not increase cGMP levels. Neurons in $\mathrm{PGE}_{1}$ contained twice as much cAMP as controls, and those grown with $\mathrm{PGE}_{1}$ and $\mathrm{CM}$ contained less cAMP but still twice as much as cultures in $\mathrm{CM}$ alone. The cGMP content of the $\mathrm{PGE}_{1}$ cultures also was increased 2- to 3-fold. Adenosine increased cAMP levels 2- to 3 -fold in both $\mathrm{L} 15-\mathrm{CO}_{2}$ medium and in $\mathrm{CM}$, and cGMP also was increased about 2 -fold. Carbachol (in the presence of hexamethonium), histamine, $\gamma$-aminobutyric acid, and bradykinin were also tested in a search for agents capable of selectively elevating cGMP, but none of these significantly altered cyclic nucleotide levels (data not shown). The most important of these observa- tions is that cholera toxin, which selectively increased only cAMP levels, was effective in decreasing the transmitter ratio. Since dbcGMP increased neuronal cAMP and since no effectors were found which selectively increased cGMP levels, the role of cGMP in transmitter choice is equivocal.

Effects of depolarization on cyclic nucleotide content. If cyclic nucleotides are second messengers for the depolarization-induced resistance to cholinergic differentiation, then elevations in cyclic nucleotides should be seen in chronically depolarized neurons. The sympathetic neurons usually do not show significant cholinergic induction by CM until after about 1 week in vitro (Patterson and Chun, 1977b; Landis, 1980). If cyclic nucleotides are involved in transmitter choice, then young neurons might show elevations in cyclic nucleotides in response to depolarization before the major developmental changes are manifested as alterations in transmitter metabolism during the 2nd and 3rd weeks (Mains and Patterson, 1973; Patterson and Chun, 1977b). To test this, transmitter synthesis and cyclic nucleotide content of neurons grown in $50 \% \mathrm{CM}$ or $20 \mathrm{mM} \mathrm{K}^{+}$were compared over a 3-week time course. The general development of these neurons will be discussed first, then the changes in cyclic nucleotide content (Figs. 1 to 3 ).

TABLE V

Effects of dibutyryl derivatives on intracellular cyclic nucleotides

Six cultures were grown in each group; two were used for assay of transmitter synthesis and four were used for assay of cyclic nucleotides and protein. All figures are for duplicate determinations except the $\mathrm{ACh} / \mathrm{CA}$ ratio for $\mathrm{R}$, which was determined on only one culture.

\begin{tabular}{|c|c|c|c|c|c|}
\hline \multicolumn{2}{|c|}{ Additives } & \multirow{2}{*}{$\mathrm{ACh} / \mathrm{CA}$} & \multirow{2}{*}{ cAMP } & \multirow{2}{*}{ cGMP } & \multirow{2}{*}{ Protein } \\
\hline Days 2-12 & Days $12-14$ & & & & \\
\hline & & & \multicolumn{2}{|c|}{$\mathrm{fmol} / \mu \mathrm{g}$} & $\mu g$ \\
\hline None & None & $0.10 \pm 0.03$ & $8.0 \pm 0.8$ & $1.1 \pm 0.1$ & $20.2 \pm 4.2$ \\
\hline $\mathrm{CM}$ & $\mathrm{CM}$ & $5.02 \pm 0.14^{a}$ & $6.0 \pm 0.1$ & $1.7 \pm 0.1$ & $19.6 \pm 2.5$ \\
\hline $\mathrm{R}$ & $\mathrm{R}$ & 0.05 & $9.8 \pm 0.8$ & $1.5 \pm 0.3$ & $19.3 \pm 2.5$ \\
\hline None & $\mathrm{dbcGMP}+\mathrm{R}$ & $0.06 \pm 0.03$ & $9.6 \pm 1.7$ & & $20.2 \pm 2.8$ \\
\hline $\mathrm{dbcGMP}+\mathrm{R}$ & dbcGMP + R & $0.20 \pm 0.02$ & $14.4 \pm 0.7^{a}$ & & $14.6 \pm 0.3$ \\
\hline $\mathrm{dbcGMP}+\mathrm{R}+\mathrm{CM}$ & $\mathrm{dbcGMP}+\mathrm{R}+\mathrm{CM}$ & $1.44 \pm 0.16^{a, b}$ & $15.4 \pm 1.4^{a}$ & & $13.8 \pm 2.8$ \\
\hline None & dbcAMP + R & $0.06 \pm 0.01$ & & $1.3 \pm 0.3$ & $22.2 \pm 1.3$ \\
\hline $\mathrm{dbcAMP}+\mathrm{R}$ & $\mathrm{dbcAMP}+\mathrm{R}$ & $0.16 \pm 0.02$ & & $1.0 \pm 0.1$ & $21.0 \pm 0.3$ \\
\hline $\mathrm{dbcAMP}+\mathrm{R}+\mathrm{CM}$ & $\mathrm{dbcAMP}+\mathrm{R}+\mathrm{CM}$ & $1.58 \pm 0.02^{a, b}$ & & $4.3 \pm 0.1^{a}$ & $16.9 \pm 1.2$ \\
\hline
\end{tabular}

${ }^{a} p<0.05$ compared to control (line 1 ).

${ }^{b} p<0.05$ compared to CM (line 2).

TABLE VI

Effects of cholera toxin, adenosine, and $P G E_{1}$

Cultures were grown in the additives shown for 14 days; $10 \mu \mathrm{M}$ RO 20-1724 was added to all cultures receiving cAMP effectors. CM was an ammonium sulfate preparation equivalent to $100 \% \mathrm{CM}$. Some cultures were assayed for cyclic nucleotides and protein as described; sister cultures were assayed for transmitter synthesis. All figures are $\pm \mathrm{SEM}$ for duplicate determinations. $\mathrm{PGE}_{1}$ and adenosine were added to $50 \mu \mathrm{M}$ and $1 \mathrm{mM}$, respectively. Cholera toxin was added at $25 \mu \mathrm{g} / \mathrm{ml}$ on days $2,6,10$, and 12 only.

\begin{tabular}{|c|c|c|c|c|c|c|}
\hline Additives & $\mathrm{ACh} / \mathrm{CA}$ & $\mathrm{ACh}$ & $\mathrm{CA}$ & cAMP & cGMP & Protein \\
\hline & & \multicolumn{2}{|c|}{ pmol/culture } & \multicolumn{2}{|c|}{$\mathrm{fmol} / \mu \mathrm{g}$} & $\mu g$ \\
\hline None & $0.2 \pm 0.0$ & $1.3 \pm 0.1$ & $8.6 \pm 0.7$ & $9.8 \pm 0.4$ & $1.6 \pm 0.1$ & $38.0 \pm 8.5$ \\
\hline $\mathrm{CM}$ & $13.5 \pm 1.1^{a}$ & $98.5 \pm 14.7^{\alpha}$ & $6.9 \pm 0.5$ & $6.3 \pm 2.1$ & $0.5 \pm 0.1$ & $77.0 \pm 7.1^{a}$ \\
\hline Cholera toxin & $<0.1$ & $<0.4$ & $10.0 \pm 2.1$ & $26.3 \pm 3.9^{a}$ & $1.7 \pm 0.8$ & $31.5 \pm 3.5$ \\
\hline Cholera toxin + CM & $2.4 \pm 0.4^{a, b}$ & $24.1 \pm 1.1^{b}$ & $10.3 \pm 1.3$ & $17.3 \pm 1.9$ & $1.0 \pm 0.3$ & $50.5 \pm 10.6$ \\
\hline $\mathrm{PGE}_{1}$ & $<0.1$ & $<0.4$ & $10.1 \pm 5.2$ & $20.3 \pm 3.3^{b}$ & $4.4 \pm 1.1^{a}$ & $33.5 \pm 0.7$ \\
\hline $\mathrm{PGE}_{1}+\mathrm{CM}$ & $3.1 \pm 0.1^{a, b}$ & $26.9 \pm 13.0^{b}$ & $8.7 \pm 3.9$ & $13.9 \pm 2.3$ & $1.3 \pm 0.3$ & $47.5 \pm 2.1$ \\
\hline Adenosine & $<0.1$ & $<0.4$ & $5.6 \pm 1.3$ & $34.2 \pm 6.5^{a}$ & $4.0 \pm 0.0$ & $28.5 \pm 7.8$ \\
\hline Adenosine + CM & $3.3 \pm 0.2^{a, b}$ & $36.7 \pm 3.3^{a, b}$ & $11.0 \pm 1.5$ & $16.8 \pm 2.0$ & $3.2 \pm 0.8^{b}$ & $54.5 \pm 3.5$ \\
\hline
\end{tabular}

${ }^{a} p<0.1$ compared to control (line 1$)$.

${ }^{b} p<0.1$ compared to CM (line 2). 
The protein content of cultures in the two media increased linearly and there were no significant differences between neurons grown in $20 \mathrm{mM} \mathrm{K}^{+}$and $\mathrm{CM}$ (Fig. 1). The development of transmitter synthesis in the two types of cultures is shown in Figure 2; the findings are compatible with previous work (Mains and Patterson, 1973; Patterson and Chun, 1977b). At 9 days, cultures in $20 \mathrm{mM} \mathrm{K}^{+}$produced significant quantities of CA, while $\mathrm{ACh}$ synthesis was barely detectable. Cultures in $50 \%$ CM made smaller, though nearly comparable, amounts of $\mathrm{CA}$ and also some ACh. The major changes in transmitter synthesis occurred during the 2nd and 3rd weeks in vitro. The neurons in $20 \mathrm{mM} \mathrm{K}^{+}$increased their ability to synthesize and store CA about 4-fold, while ACh

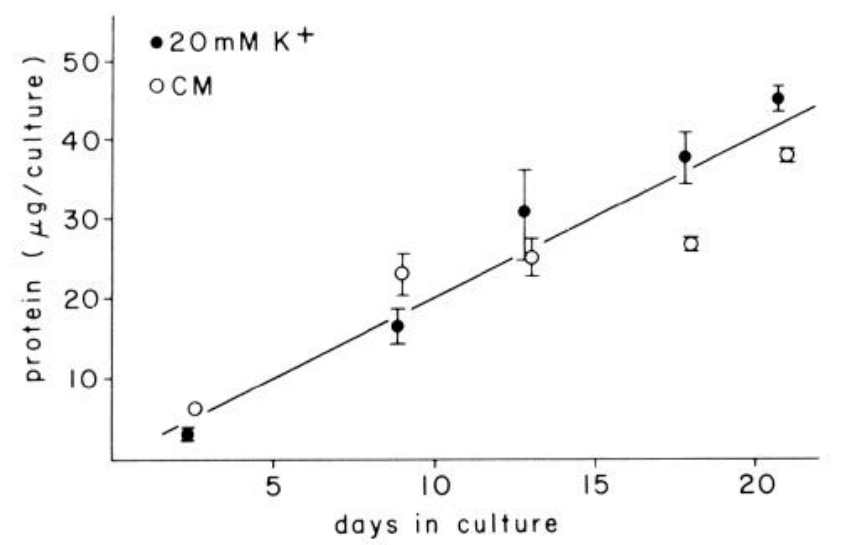

Figure 1. Effects of $\mathrm{CM}$ and $20 \mathrm{mM} \mathrm{K}^{+}$on neuronal protein content. Sister cultures were grown in $20 \mathrm{mM} \mathrm{K}^{+}$or $50 \% \mathrm{CM}$ and harvested on the days shown. Cultures were removed carefully from collagen films with a small surgical blade and assayed by the method of Lowry (Lowry et al., 1951). In Figures 1,3 , and 5 , the points represent the mean of duplicate determinations on quadruplicate cultures; bars are \pm SEM. These same samples were assayed for cAMP and cGMP to generate the data shown in Figure 3.

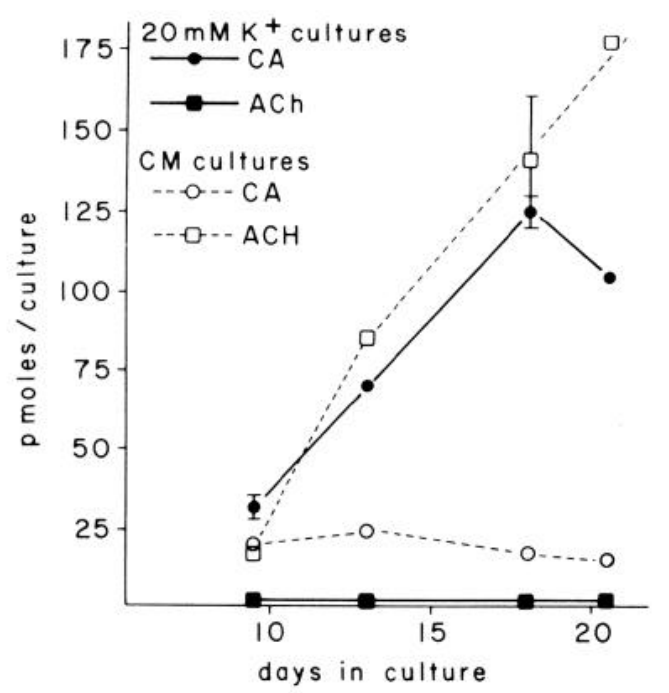

Figure 2. Effects of $\mathrm{CM}$ and $20 \mathrm{mM} \mathrm{K}^{+}$on transmitter development. Transmitter synthesis was assayed in duplicate cultures by a 4 -hr incubation with $\left[{ }^{3} \mathrm{H}\right]$ tyrosine and $\left[{ }^{3} \mathrm{H}\right]$ choline as previously described. These cultures are sister cultures to the ones used for Figures 1 and 3. synthesis remained barely detectable. Neurons grown in CM did not increase their ability to synthesize CA but did increase ACh synthesis nearly 10 -fold.

cAMP and cGMP contents were assayed on sister cultures of the ones used for assay of transmitter synthesis (Fig. 3). Both depolarized and CM-treated neurons contained more cAMP than cGMP, and the contents of both cyclic nucleotides declined roughly linearly with maturation in vitro. At all points, cAMP content was about 2-fold higher in the depolarized cultures than the CM cultures, though the absolute difference decreased with age as the content of cAMP declined. The largest absolute difference in cAMP content was measured at 3 days, when most of the neurons grown under either condition were primarily adrenergic. The difference in cGMP content between the two sets of cultures was much smaller and there was considerable overlap. Thus, chronic depolarization with $20 \mathrm{mM} \mathrm{K}^{+}$caused an increase in cAMP but not cGMP. Depolarization by veratridine (Walicke and Patterson, 1981) had similar effects on neuronal cAMP. The increase in cAMP stimulated by 20 $\mathrm{mM} \mathrm{K}^{+}$was present before the major developmental changes in transmitter content, as it should be if cAMP is the second messenger.

Combined effects of depolarization and theophylline. If cAMP is the second messenger in the developmental process stimulated by depolarization, then phosphodiesterase inhibitors should potentiate the influence of depolarization on transmitter choice. Ten micromolar isobutyl methylxanthine, RO 20-1724, or theophylline did not augment the effectiveness of elevated $\mathrm{K}^{+}$(data not shown). Higher theophylline concentrations were examined, and, surprisingly, it was observed that $2 \mathrm{~mm}$ concentrations produced cultures with significantly higher $\mathrm{ACh} / \mathrm{CA}$ ratios than controls (Table VII). This concentration of theophylline is somewhat toxic and resulted in a lower protein content. The neurons also appear unhealthy with phase microscopy, and the cultures have a tendency to roll up and occasionally detach from the dish. Despite its toxicity, theophylline appeared to en-

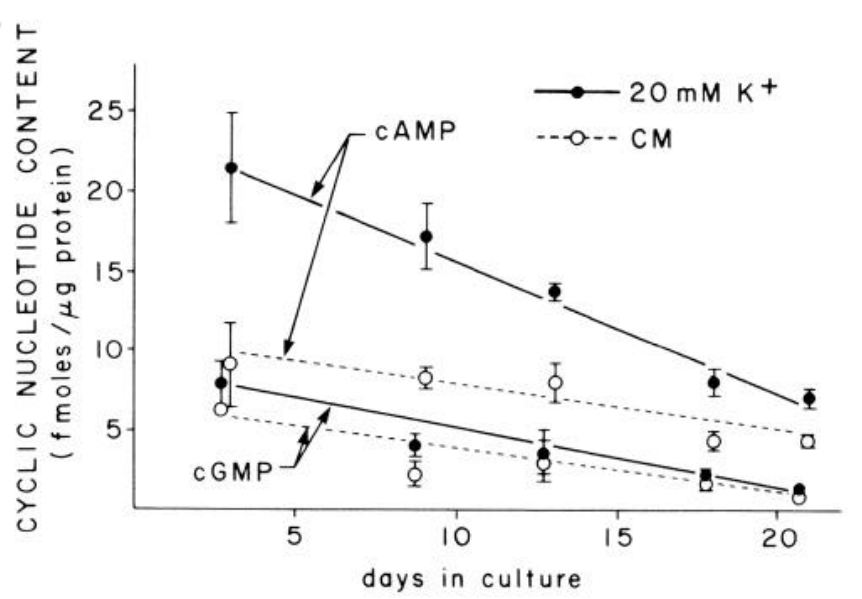

Figure 3. Developmental changes in cyclic nucleotide content. Duplicate cultures were harvested on the days shown, combined, and assayed for cAMP and cGMP as previously described. Each point represents duplicate determinations on material from both cultures. The same samples were assayed for protein content (Fig. 1). 
TABLE VII

Effects of theophylline

All cultures were grown for 2 weeks in $50 \% \mathrm{CM}$ and the additives shown. In this table, $\mathrm{K}$ refers to $20 \mathrm{~mm} \mathrm{~K}^{+}$and Theo refers to $2 \mathrm{~mm}$ theophylline. Assays were as previously described. All figures represent duplicate determinations.

\begin{tabular}{|c|c|c|c|c|c|}
\hline Additives & cAMP & $\mathrm{ACh} / \mathrm{CA}$ & $\mathrm{ACh}$ & $\mathrm{CA}$ & Protein \\
\hline & $\mathrm{fmol} / \mu \mathrm{g}$ & & \multicolumn{2}{|c|}{ pmol/culture } & $\mu g$ \\
\hline $\mathrm{CM}$ & $5.1 \pm 0.2$ & $5.9 \pm 0.3$ & $70.5 \pm 19.8$ & $11.8 \pm 2.7$ & $56.7 \pm 3.3$ \\
\hline $\mathrm{CM}+\mathrm{K}$ & $7.4 \pm 0.1^{a}$ & $0.4 \pm 0.2$ & $5.2 \pm 1.7^{a}$ & $14.9 \pm 2.0$ & $48.1 \pm 1.3^{b}$ \\
\hline $\mathrm{CM}+$ Theo & $11.2 \pm 0.8^{a, c}$ & $20.9 \pm 4.2^{a, c}$ & $28.3 \pm 0.3^{b}$ & $1.4 \pm 0.3^{a, c}$ & $25.7 \pm 0.7^{a, c}$ \\
\hline $\mathrm{CM}+\mathrm{K}+$ Theo & $9.8 \pm 0.0^{a, c}$ & $6.6 \pm 0.3$ & $18.7 \pm 0.4^{a}$ & $2.8 \pm 0.2^{a, c}$ & $22.6 \pm 0.8^{a_{, c}}$ \\
\hline
\end{tabular}

${ }^{a} p<0.05$ compared to control (line 1 ).

${ }^{b} p<0.1$ compared to control (line 1$)$.

c $p<0.05$ compared to $\mathrm{K}$ (line 2 ).

hance cholinergic differentiation and to inhibit the effect of $\mathrm{K}^{+}$depolarization on transmitter choice. Since theophylline inhibited the effects of $\mathrm{K}^{+}$on transmitter choice, its effects on cAMP content were examined also (Table VII). In this experiment, depolarization increased the cAMP content by 1.5 -fold and lowered the $\mathrm{ACh} / \mathrm{CA}$ ratio more than 10-fold. Cells grown in theophylline and CM, with or without $\mathrm{K}^{+}$, contained significantly more cAMP (per $\mu \mathrm{g}$ of protein) than cultures in $\mathrm{K}^{+}$alone but had $\mathrm{ACh} / \mathrm{CA}$ ratios comparable to that of cultures in $\mathrm{CM}$ alone. Thus, theophylline differs from the other agents described thus far in that it elevated cAMP but enhanced cholinergic induction. It also inhibited the developmental effect of depolarization while further augmenting the cAMP increase.

Since the levels of cAMP seen in young neurons are probably more important for development than those seen in the mature neurons, a developmental study was done to verify that theophylline also increases cAMP in younger neurons. The development of transmitter synthesis and cyclic nucleotide content in cultures grown in $\mathrm{CM}, 20 \mathrm{mM} \mathrm{K}^{+}+\mathrm{CM}$, or $2 \mathrm{mM}$ theophylline $+20 \mathrm{mM}$ $\mathrm{K}^{+}+\mathrm{CM}$ are compared in Figures 4 and 5 . The ACh/CA ratios in the CM cultures increased over 2 weeks, while those in the depolarized cultures remained low. Cultures kept in theophylline as well as $20 \mathrm{mM} \mathrm{K} \mathrm{K}^{+}$expressed similar, though slightly lower, $\mathrm{ACh} / \mathrm{CA}$ ratios than those in $\mathrm{CM}$ alone. Thus, theophylline suppressed the effect of $20 \mathrm{mM} \mathrm{K}^{+}$at all ages tested. On the other hand, the cultures in theophylline and those in $20 \mathrm{mM} \mathrm{K}^{+}$clearly had higher levels of cAMP than the CM cultures at early ages. As seen in the other experiments, cAMP levels declined with time, so that, by 14 days, there was less absolute difference among the three groups. Thus, theophylline, like depolarization, increased cAMP in young neurons, but, unlike depolarization, theophylline produced cholinergic cultures.

\section{Discussion}

Effects of exogenous cyclic nucleotides on transmitter choice. Addition of dbcAMP, dbcGMP, adenosine, $\mathrm{PGE}_{1}$, or cholera toxin decreases the amount of ACh synthesized and lowers the $\mathrm{ACh} / \mathrm{CA}$ ratio. These changes occurred with relatively little effect on neuronal survival or overall growth as measured by protein content. Thus, cyclic nucleotides might be developmental signals which decrease the neuronal response to the cholinergic factor

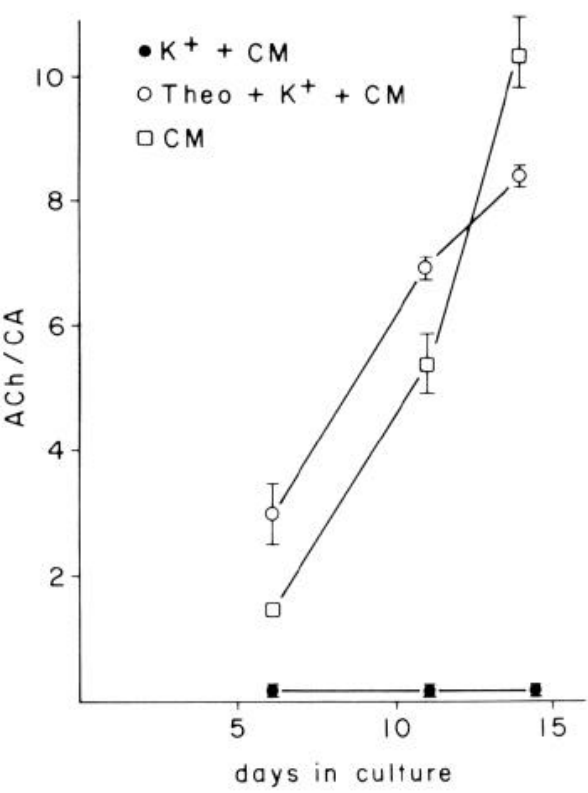

Figure 4. Effect of theophylline (Theo) on transmitter development. All cultures were grown in $50 \% \mathrm{CM}$; some were exposed also to $20 \mathrm{mM} \mathrm{K}^{+}$or $20 \mathrm{mM} \mathrm{K}^{+}$and $2 \mathrm{~mm}$ theophylline as indicated. On days 6,11 , and 14 , duplicate cultures from each group were assayed for transmitter synthesis as previously described.

in $\mathrm{CM}$ and allow the neurons to complete adrenergic differentiation.

It is possible, however, that changes in the $\mathrm{ACh} / \mathrm{CA}$ ratio reflect modulation of the activity of existing transmitter synthesizing enzymes rather than alterations in neuronal development. There is abundant evidence that cAMP can modulate acutely neurotransmitter metabolism, including the phosphorylation and activation of TH (Joh et al., 1978; Vulliet et al., 1980).

Exposing the neurons to dibutyryl derivatives for 2 days did not alter the amounts of ACh and CA synthesized, and removing the derivatives from the medium for 2 days before assay did not reverse the decrease in the $\mathrm{ACh} / \mathrm{CA}$ ratio. It appears, therefore, that stable alterations in transmitter choice, most likely alterations in the content of transmitter synthesizing enzymes, are occurring. This interpretation is consistent with previous reports in which cAMP derivatives increase levels of TH and dopamine $\beta$-hydroxylase in neuroblastoma cells (Prasad, 1975; Waymire et al., 1978) and organ-cultured 


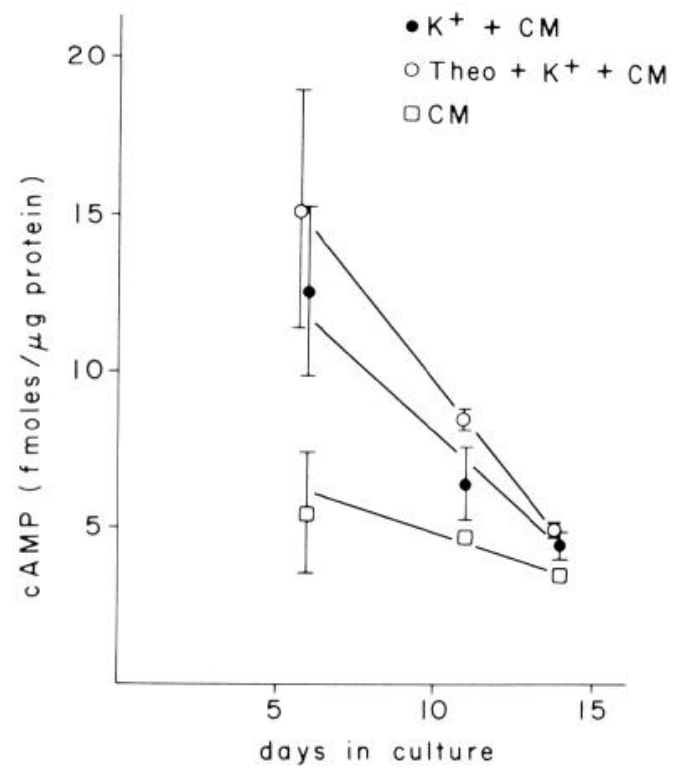

Figure 5. Effect of theophylline (Theo) on cyclic nucleotide content. Sister cultures of the ones used in Figure 4 were used for assay of cyclic nucleotide and protein content. Quadruplicate cultures were used for duplicate determinations of protein and cAMP as previously described.

sympathetic ganglia (Goodman et al., 1974; Keen and McLean, 1974; Mackay and Iversen, 1972). It should be noted also that transmitter synthesis was always determined in a 4-hr incubation in the absence of cyclic nucleotides and phosphodiesterase inhibitors. Since acutely increased $\mathrm{TH}$ activity generally returns to base line in approximately $30 \mathrm{~min}$ (Salzman and Roth, 1980; Weiner et al., 1977), such effects are likely to be reversed early in the incubation period. The results of short exposures to cyclic nucleotide derivatives do not show that phosphorylation-mediated activation of $\mathrm{TH}$ does not occur in these neurons, only that it is probably not the phenomenon under study in the present experiments.

The effect of cyclic nucleotides on transmitter choice is analogous to the developmental effect of depolarization and fulfills the first of the criteria for demonstration that cyclic nucleotides function as second messengers in depolarization. Although the effects are qualitatively similar, they differ quantitatively. Depolarization with $20 \mathrm{~mm}$ $\mathrm{K}^{+}$or veratridine lowers the $\mathrm{ACh} / \mathrm{CA}$ ratio in CM-induced neurons as much as 500-fold (Walicke et al., 1977), while the cyclic nucleotide agents cause alterations of 3to 15 -fold. Although care was taken to optimize conditions under which neurons were exposed to dbcAMP, dbcGMP, adenosine, and $\mathrm{PGE}_{1}$, it is possible that unknown factors limited their effectiveness. Since butyrate enhances cholinergic differentiation, it is possible that the results with dibutyryl derivatives represent a balance between opposing influences of butyrate and cyclic nucleotides. The effectiveness of adenosine and $\mathrm{PGE}_{1}$ could be limited by receptor desensitization (Ayad and Burns, 1979). The data do indicate, however, that these exogenous cyclic nucleotide derivatives are less effective than depolarization in influencing transmitter choice.

Since cyclic nucleotides have qualitatively the same effects on neuronal development as depolarization, the possibility that these agents are depolarizing the cells must be considered. McAfee and Greengard (1972) observed that dbcAMP hyperpolarizes sympathetic neurons of the rabbit, while dbcGMP depolarizes them. Subsequent studies of the effect of cAMP on membrane potential have demonstrated hyperpolarization (Brown et al., 1979) or no alteration in resting potential (Busis et al., 1978; Dun and Karczmar, 1977; Gallagher and Shinnick-Gallagher, 1977). Depolarization by cAMP was reported by Gallagher and Shinnick-Gallagher (1977) after massive intracellular injection but not in response to extracellular application. Depolarization by dbcGMP has been observed also (Busis et al., 1978; Dun et al., 1978), though again, no effect has also been reported (Weight et al., 1978). Adenosine has been reported to hyperpolarize rat sympathetic neurons in one study (Brown et al., 1979 ) and to depolarize them in another (Gallagher and Shinnick-Gallagher, 1977). $\mathrm{PGE}_{1}$ has been observed to influence transmitter release (Westfall, 1977), but it has not been reported to cause any alteration in membrane potential (McAfee and Greengard, 1972). Since several of these agents appear to have no effect on membrane potential or to cause hyperpolarization, it seems unlikely that the alteration in development by all of these agents is mediated by depolarization.

In the present study, both dbcAMP and dbcGMP act to maintain adrenergic differentiation. This could be interpreted as evidence that both cAMP and cGMP have the same effect on neuronal transmitter choice. Results based on observations with dibutyryl analogues must be taken with care, however, since the specificity of the cAMP and cGMP analogues for their respective cyclic nucleotide-dependent protein kinases is reduced (Kuo et al., 1976; Miyamoto et al., 1969). Furthermore, as previously observed in neuroblastoma cell lines (Prasad and Gilmer, 1974; Zwiller et al., 1977), prolonged exposure of primary sympathetic neurons to the dibutyryl derivative of one nucleotide leads to increases in the intracellular levels of the other. Thus, it is possible that dbcAMP acts by increasing intracellular cGMP or vice versa. The results with the effectors capable of increasing intracellular cyclic nucleotides help to clarify the situation. Cholera toxin maintains neuronal adrenergic differentiation and specifically increases only intracellular cAMP, suggesting that increased cAMP alone is sufficient to direct the neurons toward adrenergic development. A search was made for agents which specifically elevate cellular cGMP, but none was found. Since dbcGMP increases intracellular cAMP and since an increase in cAMP alone is correlated with adrenergic development, it is possible that dbcGMP is really acting through cAMP. The results do not exclude the possibility that cGMP has similar effects on transmitter development, but all of the observed effects can be ascribed to cAMP alone.

These studies also yielded another agent with interesting effects on transmitter choice, butyrate, which suppresses CA synthesis and favors cholinergic differentiation. Although butyrate increases TH levels in some neuroblastoma cell lines (Lloyd et al., 1978), its effect on the primary sympathetic neurons is the opposite of the cyclic nucleotides. Further study of this developmental effect of butyrate could be helpful in illuminating the cellular mechanisms underlying transmitter choice. It 
has been suggested recently that inhibition of histone deacetylation may mediate some of the cellular effects of butyrate (Candido et al., 1978; Sealy and Chalkley, 1978).

Depolarization and intracellular cAMP. Like most cells, the sympathetic neurons contain considerably more cAMP than cGMP. The cyclic AMP content is comparable to the amount previously observed in isolated chick sympathetic neurons by Wallace et al. (1978). Depolarization with $20 \mathrm{~mm} \mathrm{~K} \mathrm{~K}^{+}$increases the neuronal content of cAMP 1.5- to 2-fold. The levels of cGMP are usually either unaffected or slightly lowered by depolarization. These observations fulfill the second criterion for establishing cAMP as a second messenger. This difference in cAMP content between depolarized and CM cultures can be demonstrated at early ages before there are significant differences in transmitter content. The difference remains constant at about 2 -fold over several weeks but is quantitatively greatest at early times and becomes smaller as the cultures mature and fully express either their adrenergic or cholinergic character. The third criterion, that increases in cAMP should precede changes in transmitter content, therefore, is fulfilled.

Theophylline, cAMP, and depolarization. If the developmental effects of depolarization are mediated by cAMP, then addition of phosphodiesterase inhibitors would be expected to suppress further cholinergic induction. Addition of theophylline to cultures depolarized with elevated $\mathrm{K}^{+}$does increase further the content of cAMP; however, it directs the neurons toward cholinergic development. Theophylline also increases cAMP in young neurons which have not yet undergone the final enhancement of transmitter function. Thus, theophylline appears to potentiate the effect of depolarization on cAMP accumulation but reverses its effects on neuronal development.

Theophylline is recognized to have several different effects on neurons: (i) It inhibits phosphodiesterase, (ii) it blocks adenosine receptors (Sattin and Rall, 1970), (iii) it alters membrane potential and ionic conductances (Busis et al., 1978; Dun and Karczmar, 1977), and (iv) it releases $\mathrm{Ca}^{2+}$ from intracellular stores resembling the sarcoplasmic reticulum (Johnson and Inesi, 1969). The increase in cAMP is probably due to the activity of theophylline as a phosphodiesterase inhibitor. Which of the effects of theophylline is responsible for inhibiting the effects of depolarization on development is uncertain but is discussed at greater length in the accompanying paper (Walicke and Patterson, 1981). It should be noted that the concentrations of theophylline employed are somewhat toxic. Despite a decline in protein content, the neurons continue to make neurotransmitters and cyclic nucleotides in quantities roughly comparable to controls. Simple toxicity does not in itself favor cholinergic differentiation, since veratridine and elevated $\mathrm{CaCl}_{2}$, which are also toxic, favor adrenergic development (Walicke et al., 1977; Walicke and Patterson, 1981).

In summary, several lines of evidence presented here favor cAMP as a second messenger for the effects of depolarization or activity on transmitter choice. Closer examination, however, reveals some inconsistencies in the evidence. Exogenous cyclic nucleotide derivatives and agents which elevate intracellular cAMP are much less effective than depolarization in lowering the ACh/
CA ratio. The phosphodiesterase inhibitor, theophylline, is able to increase the cAMP levels in depolarized cultures even while reversing the effects on transmitter choice. In the following paper (Walicke and Patterson, 1981), other agents which can uncouple the increase in cAMP from subsequent changes in transmitter choice will be discussed also. Thus, although cAMP can influence the transmitter choice under some circumstances, there is evidence inconsistent with the hypothesis that it is the second messenger in the developmental effects of activity.

\section{References}

Ayad, S., and G. Burns (1979) Prostaglandin $\mathrm{E}_{1}$-induced desensitization of prostaglandin-sensitive adenylate cyclase of cultured mammalian cells. Exp. Cell Res. 118: 229-235.

Brown, D., M. Caulfield, and P. Kirby (1979) Relation between catecholamine-induced cyclic AMP changes and hyperpolarization in isolated rat sympathetic ganglia. J. Physiol. (Lond.) 290: 441-451.

Bunge, R., M. Johnson, and C. Ross (1978) Nature and nurture in development of the autonomic neuron. Science 199: 14091416.

Busis, N., F. Weight, and P. Smith (1978) Synaptic potentials in sympathetic ganglia: Are they mediated by cyclic nucleotides? Science 200: 1079-1081.

Candido, F., R. Reeves, and J. Davie (1978) Sodium butyrate inhibits histone deacetylation in cultured cells. Cell 14: 105113.

Dun, N., and A. Karczmar (1977) A comparison of the effect of theophylline and cyclic adenosine $3^{\prime}: 5^{\prime}$-monophosphate on the superior cervical ganglion of the rabbit by means of the sucrose-gap method. J. Pharmacol. Exp. Ther. 202: 89-96.

Dun, N., K. Kaibara, and A. Karczmar (1978) Muscarinic and cGMP-induced membrane potential changes: Differences in electrogenic mechanisms. Brain Res. 150: 658-661.

Gallagher, J., and P. Shinnick-Gallagher (1977) Cyclic nucleotides injected intracellularly into rat superior cervical ganglion cells. Science 198: 851-852.

Goodman, R., F. Oesch, and H. Thoenen (1974) Changes in enzyme patterns produced by high potassium concentration and dibutyryl cyclic AMP in organ cultures of sympathetic ganglia. J. Neurochem. 23: 369-378.

Guidotti, A., and E. Costa (1977) Trans-synaptic regulation of tyrosine 3-mono-oxygenase biosynthesis in rat adrenal medulla. Biochem. Pharmacol. 26: 817-823.

Hawrot, E., and P. H. Patterson (1978) Long-term culture of dissociated sympathetic neurons. Methods Enzymol. 58: 574583.

Joh, T., D. Park, and D. Reis (1978) Direct phosphorylation of tyrosine hydroxylase by cAMP-dependent protein kinase: Mechanism of enzyme activation. Proc. Natl. Acad. Sci. U. S. A. 75 : $4744-4748$.

Johnson, P., and G. Inesi (1969) The effect of methylxanthines and local anaesthetics on fragmented sarcoplasmic reticulum. J. Pharmacol. Exp. Ther. 169: 308-314.

Keen, P., and W. McLean (1974) Effect of dibutyryl-cyclic AMP and dexamethasone on noradrenaline synthesis in isolated superior cervical ganglia. J. Neurochem. 22: 5-10.

Kuo, J., W. Kuo, M. Shoji, S. Davis, V. Seery, and T. Donnelly (1976) Purification and general properties of guanosine $3^{\prime}: 5^{\prime}$ monophosphate-dependent protein kinase from guinea pig fetal lung. J. Biol. Chem. 251: 1759-1766.

Landis, S. (1980) Developmental changes in the neurotransmitter properties of dissociated sympathetic neurons: A cytochemical study of the effects of medium. Dev. Biol. 77: 349361 .

Lloyd, T., B. Ebersole, and F. Schneider (1978) Stimulation of 
tyrosine hydroxylase activity in cultured mouse neuroblastoma cells by monocarboxylic acids. J. Neurochem. 30: 16411643.

Lowry, O., N. Rosebrough, A. Farr, and R. Randall (1951) Protein measurement with the Folin phenol reagent. J. Biol. Chem. 193: 265-275.

Mackay, A., and L. Iversen (1972) Increased tyrosine hydroxylase activity of sympathetic ganglia cultured in the presence of dibutyryl cyclic $\Lambda$ MP. Brain Res. 48: 424-426.

Mains, R., and P. Patterson (1973) Primary cultures of dissociated sympathetic neurons. III. Changes in metabolism with age in culture. J. Cell Biol. 59: 361-366.

McAfee, D., and P. Greengard (1972) Adenosine 3',5'-monophosphate: Electrophysiological evidence for a role in synaptic transmission. Science 178: 300-312.

Miyamoto, E., J. Kuo, and P. Greengard (1969) Cyclic nucleotide-dependent protein kinases. III. Purification and properties of adenosine $3^{\prime}: 5^{\prime}$-monophosphate-dependent protein kinase from bovine brain. J. Biol. Chem. 244: 6395-6402.

Patterson, P. (1978) Environmental determination of autonomic neurotransmitter functions. Annu. Rev. Neurosci. 1: 1-17.

Patterson, P., and L. Chun (1977a) The induction of acetylcholine synthesis in primary cultures of dissociated rat sympathetic neurons. I. Effects of conditioned medium. Dev. Biol. 56: 263-280.

Patterson, P., and L. Chun (1977b) The induction of acetylcholine synthesis in primary cultures of dissociated rat sympathetic neurons. II. Developmental aspects. Dev. Biol. 60: 473481.

Prasad, K. (1975) Differentiation of neuroblastoma cells in culture. Biol. Rev. 50: 129-165.

Prasad, K., and K. Gilmer (1974) Demonstration of dopaminesensitive adenylate cyclase in malignant neuroblastoma cells and change in sensitivity of adenylate cyclase to catecholamines in "differentiated" cells. Proc. Natl. Acad. Sci. U. S. A. $71: 2525-2529$.

Reichardt, L., and P. Patterson (1977) Neurotransmitter synthesis and uptake by isolated sympathetic neurons in microcultures. Nature 270: 147-151.

Salzman, P., and R. Roth (1980) Poststimulation catecholamine synthesis and tyrosine hydroxylase activation in central noradrenergic neurons. II. Depolarized hippocampal slices. J. Pharmacol. Exp. Ther. 212: 74-84.

Sattin, A., and T. Rall (1970) The effect of adenosine and adenine nucleotides on the cyclic adenosine $3^{\prime}, 5^{\prime}$-phosphate content of guinea pig cerebral cortex slices. Mol. Pharmacol. 6: $13-23$.

Scheffé, H. (1959) Analysis of Variance, John Wiley, New York.

Sealy, L., and R. Chalkley (1978) The effect of sodium butyrate on histone modification. Cell 14: 115-121.

Vulliet, P., T. Langan, and N. Weiner (1980) Tyrosine hydroxylase: A substrate of cyclic AMP-dependent protein kinase. Proc. Natl. Acad. Sci. U. S. A. 77: 92-96.

Walicke, P. A., and P. H. Patterson (1981) On the role of $\mathrm{Ca}^{2+}$ in the transmitter choice made by cultured sympathetic neurons. J. Neurosci. 1: 343-350.

Walicke, P., R. Campenot, and P. Patterson (1977) Determination of transmitter function by neuronal activity. Proc. Natl. Acad. Sci. U. S. A. 74: 5767-5771.

Wallace, L., L. Partlow, and J. Ferrendelli (1978) Comparison of levels of adenosine- $3^{\prime}, 5^{\prime}$-monophosphate in highly purified and mixed cultures of neurons and non-neuronal cells from embryonic chick sympathetic ganglia. J. Neurochem. 31: 801807.

Waymire, J., K. Gilmer-Waymire, and R. Boehme (1978) Concomitant elevation of tyrosine hydroxylase and dopamine beta-hydroxylase by cyclic AMP in cultured mouse neuroblastoma cells. J. Neurochem. 31: 699-705.

Weight, F., P. Smith, and J. Schulman (1978) Postsynaptic potential generation appears independent of synaptic elevations of cyclic nucleotides in sympathetic neurons. Brain Res. 158: 197-202.

Weiner, N., F. Lee, E. Barnes, and E. Dreyer (1977) Enzymology of tyrosine hydroxylase and the role of cyclic nucleotides in its regulation. In Structure \& Function of Monoamine Enzymes, E. Usdin, N. Weiner, and M. Youdin, eds., pp. 109148, Marcel Dekker, Inc., New York.

Westfall, T. (1977) Local regulation of adrenergic neurotransmission. Physiol. Rev. 57: 659-728.

Zigmond, R., and A. Chalazonitis (1979) Long-term effects of preganglionic nerve stimulation on tyrosine hydroxylase activity in rat superior cervical ganglion. Brain Res. 164: 137152.

Zwiller, J., C. Goridis, J. Ciesielski-Treska, and P. Mandel (1977) Cyclic GMP in a neuroblastoma clone: Possible involvement in morphological differentiation induced by dibutyryl cyclic AMP. J. Neurochem. 29: 273-278. 